\section{Eficácia das vacinas comercialmente disponíveis contra a infecção pelo papilomavírus em mulheres: revisão sistemática e metanálise}

\author{
Efficacy of commercially available vaccines against \\ HPV infection in women: a systematic review and \\ meta-analysis
}

\section{Eficacia de las vacunas disponibles en el mercado contra la infección por papilomavirus en mujeres: revisión sistemática y metaanálisis}

\footnotetext{
1 Secretaria Municipal de Saúde e Defesa Civil do Rio de Janeiro, Rio de Janeiro, Brasil.

2 Instituto de Medicina Social, Universidade do Estado do Rio de Janeiro, Rio de Janeiro, Brasil.

${ }^{3}$ Escola Nacional de Saúde Pública Sergio Arouca,

Fundação Oswaldo Cruz, Rio de Janeiro, Brasil.

${ }^{4}$ Faculdade de Enfermagem, Universidade do Estado do Rio de Janeiro, Rio de Janeiro Brasil.

Correspondência S. C. F. Araujo Secretaria Municipal de Saúde e Defesa Civil do Rio de Janeiro.

Estrada do Monteiro 323, bloco 2, apto. 210, Rio de Janeiro, RJ 23046-830, Brasil. silvia.araujo@smsdc.rio. rj.gov.br
}

\begin{abstract}
Persistent HPV infection is a necessary condition for the occurrence of cervical cancer. Prophylactic HPV vaccines have been developed to reduce the incidence of cervical cancer. Two vaccines are commercially available: bivalent (types 16, 18) and quadrivalent (6, 11, 16 and 18). This study aimed to perform a systematic review and metaanalysis of the HPV vaccines' efficacy in women, focusing its performance stratified by clinical outcomes. Randomized controlled trials (RCT) published between 2000 and 2009 were identified from searches of MEDLINE, LILACS and Cochrane Library, and evaluated by two independent reviewers. Six RCT were selected. The vaccines reduced the risk of precursor lesions of cervical cancer, presenting efficacy of 97\% (95\%CI: 90-99) for CIN 2 and 96\% (95\%CI: 89-99) for CIN 3 , in the per protocol analysis. The efficacies in the analysis by intention to treat were smaller: 63\% (95\%CI: 52-71) and 42\% (95\%CI: 26-55), respectively. In order to evaluate its effectiveness on the incidence and mortality rates for cervical cancer, longer-term studies will be needed.
\end{abstract}

Papillomavirus Vaccines; Uterine Cervical Neoplasms; Efficacy; Health Technology Evaluation
Silvia Cristina Fonseca de Araujo ${ }^{1}$

Rosângela Caetano ${ }^{2}$

Jose Ueleres Braga 2,3

Frances Valéria Costa e Silva 4

\section{Resumo}

Infecção persistente por HPV é condição necessária para ocorrência de câncer do colo de útero. Visando a reduzir sua incidência, foram desenvolvidas vacinas profiláticas contra HPV, existindo duas formulações comercialmente disponíveis: bivalente (subtipos 16 e 18) e quadrivalente (6, 11, 16 e 18). Realizou-se uma metanálise da eficácia dessas vacinas em mulheres, com foco na avaliação estratificada por desfechos clínicos. Ensaios clínicos randomizados (ECR) publicados entre 2000 e 2009 foram identificados com base em busca no MEDLINE, Biblioteca Cochrane e LILACS, e avaliados por dois revisores independentes. Seis ECR foram incluídos na metanálise. As vacinas reduziram o risco de ocorrência de lesões precursoras da neoplasia, com eficácia de 97\% (IC95\%: 90-99) para NIC 2 e 96\% (IC95\%: 89-99) para NIC 3, nas análises por protocolo. As eficácias nas análises por intenção de tratar foram menores: 63\% (IC95\%: 52-71) e 42\% (IC95\%: 26-55), respectivamente. Para avaliação de sua eficácia sobre a incidência e mortalidade por câncer do colo de útero são necessários estudos com maior tempo de seguimentos.

Vacinas contra Papillomavírus; Neoplasias do Colo de Útero; Eficácia; Avaliação de Tecnologias de Saúde 


\section{Introdução}

O câncer do colo de útero é ainda um importante problema de saúde pública, particularmente nas regiões mais pobres. Anualmente, surgem cerca de 500 mil novos casos e perto de 230 mil mulheres morrem, em todo o mundo, acometidas pela neoplasia 1 . Mais de $80 \%$ dos casos ocorrem nos países em desenvolvimento 2 . No Brasil, a incidência estimada para 2013 é de 17.540 novos casos, com um risco de 17 casos/100 mil mulheres 3 .

Estudos confirmam a presença do papilomavírus humano (HPV) em quase $100 \%$ dos casos desses cânceres 4 . Cerca de $20 \%$ de indivíduos sadios, em todo o mundo, estão infectados pelo HPV 5. A maior parte dessas infecções é assintomática e transitória, tornando-se completamente indetectável dentro de um a dois anos 6 , mas a infecção persistente pelo vírus favorece o desenvolvimento de lesões pré-cancerosas e, posteriormente, da neoplasia.

A detecção precoce de lesões precursoras com o uso do teste de Papanicolaou é a principal estratégia de combate dessa neoplasia, mas o rastreamento populacional não afeta a incidência de infecção pelo HPV, e as lesões necessitam de seguimento cuidadoso e tratamento. Devido à baixa sensibilidade do teste, $50 \%$ dos adenocarcinomas e $25 \%$ dos carcinomas escamosos ocorrem em mulheres examinadas, mesmo em países com programa de rastreamento adequado 7 .

Vacinas profiláticas contra o HPV foram desenvolvidas a partir de 1993, objetivando reduzir a infecção e incidência do câncer do colo de útero. Parecem induzir títulos de anticorpos substancialmente mais elevados do que aqueles que acompanham a imunidade natural. A partir de 2006, começaram a ser comercializadas; mais de cem países já aprovaram essas vacinas para uso, com diversos deles incluindo o financiamento da imunização em seus sistemas de saúde 8 , sendo relevante a presença de informação atualizada e qualificada para esses processos de decisão.

Atualmente, duas vacinas encontram-se comercialmente disponíveis para utilização: a vacina bivalente, que protege contra os tipos virais 16 e 18, e a quadrivalente, que oferece proteção contra os tipos $6,11,16$ e 18, que protegeria adicionalmente para condilomas anogenitais associados à infecção pelos subtipos 6 e 11 .

São eficazes para os tipos virais incluídos em sua formulação, permanecendo o risco de infecção por outros tipos oncogênicos, que correspondem a 30\% dos casos de câncer 9,10, dependendo da prevalência dos diversos subtipos virais em cada região e país.
A vacina não é terapêutica nem eficaz em mulheres com infecção no momento da vacinação 11, reforçando a necessidade de que seja aplicada na pré-adolescência e adolescência. Mulheres sexualmente ativas podem até se beneficiar, mas apenas para a proteção contra subtipos que ainda não tenham sido adquiridos.

Estudos sobre eficácia das vacinas são escassos e pouco se sabe sobre seu impacto em termos de prevenção dos casos de câncer invasivo. A eficácia tem sido avaliada considerando desfechos intermediários, devido ao tempo relativamente pequeno de seguimento das populações incluídas nos ensaios clínicos em relação à história natural da neoplasia, reconhecidamente longa e com aspectos ainda não plenamente compreendidos.

Já existem algumas revisões sistemáticas sobre o tema $12,13,14$, que sinalizam para a eficácia da vacinação profilática em mulheres sem exposição prévia aos subtipos virais. Contudo, alguns delas incluíram produtos vacinais não comercializados, como a vacina monovalente, não exploraram potenciais diferenciais de eficácia segundo faixa etária e tenderam a focar em um espectro estreito de desfechos intermediários. Estudos primários com maior tempo de seguimento, publicados mais recentemente, não foram contemplados nessas revisões anteriores.

Nesse cenário de poucos estudos primários e revisões sistemáticas sobre a eficácia vacinal e de dificuldade em analisar os resultados devido às distintas faixas etárias e desfechos estudados, este trabalho objetivou revisar sistematicamente a literatura sobre a eficácia das vacinas comercialmente disponíveis contra o HPV, em mulheres, privilegiando uma avaliação estratificada pelos tipos de desfechos.

\section{Método}

\section{Identificação e seleção de estudos}

Foi realizada uma busca nas bases de referências bibliográficas MEDLINE (via PubMed), LILACS (via Biblioteca Virtual de Saúde) e Biblioteca Cochrane (via Biblioteca Virtual de Saúde), visando a localizar ensaios clínicos controlados randomizados que avaliassem a eficácia das vacinas bivalente e quadrivalente contra a infecção pelo HPV em mulheres. A busca foi realizada no período de janeiro de 2000, que corresponde ao início da década de comercialização da vacina, a novembro de 2009.

Estratégias de busca compatíveis com os descritores de cada base pesquisada foram delineadas com o auxílio de um profissional de bibliote- 
conomia. Essas estratégias incluíram a pesquisa de descritores ou de palavras no texto relacionados à doença [uterine cervical neoplasms, cervical intraepithelial neoplasia, uterine cervical diseases, uterine cervical dysplasia, cervix neoplasms, cervical neoplasms, cervical cancer], ao agente infeccioso [Papillomaviridae, papillomavirus infections, human papillomavirus, papillomavirus] e ao tipo de intervenção [viral vaccines, cancer vaccines, vaccines, vaccination]. A essa busca, foram adicionados filtros de elevada sensibilidade para estudos terapêuticos 15. As estratégias de busca utilizadas podem ser consultadas a partir de correspondência aos autores.

A busca limitou-se a humanos. Foi realizada sem qualquer restrição de idioma, embora posteriormente na fase de seleção se incluíssem apenas estudos publicados em português, inglês e espanhol. Esse processo permitiu a identificação de eventuais publicações não incluídas por conta do idioma.

A inclusão teve como base os seguintes critérios: estudos que avaliavam a eficácia das vacinas bivalente e quadrivalente contra a infecção pelo HPV; utilizando placebo ou outras vacinas como controle; incluindo somente mulheres, sem restrição de idade, raça ou contato prévio com o vírus, e tendo como desfechos a resposta imune, infecção persistente pelo HPV ou lesões precursoras do câncer do colo de útero.

Foram critérios de exclusão: editoriais, cartas, artigos de revisão, resumos de conferências; estudos em animais; trabalhos que incluíam apenas indivíduos do sexo masculino ou vacinas não comercialmente disponíveis, como as monovalentes.

Tanto a avaliação de títulos e resumos quanto de texto completo foram realizadas por dois revisores (S.C.F.A e F.V.C.S.), de forma independente, com as discordâncias examinadas e resolvidas por um terceiro revisor (R.C.).

Revisões e textos selecionados para leitura completa foram examinados na tentativa de recuperar artigos relevantes não identificados pelas estratégias de busca.

As referências bibliográficas identificadas por meio da estratégia de busca foram gerenciadas pelo software EndNote, versão X (Thomson Research Soft., Carlsbad, Estados Unidos).

\section{Avaliação de qualidade metodológica}

A qualidade metodológica foi avaliada de forma independente e por dois revisores (S.C.F.A. e F.V.C.S.), utilizando o checklist CONSORT 16 para avaliação dos ensaios clínicos controlados randomizados. Desse modo, todos os ensaios clínicos incluídos foram examinados no que se refere aos seguintes critérios de qualidade: justificativa para o estudo; descrição de objetivos específicos e hipóteses; critérios de elegibilidade dos pacientes; descrição da intervenção; descrição dos desfechos primário e secundário; justificativa do tamanho da amostra; detalhamento dos procedimentos de mascaramento e randomização.

\section{Extração de dados}

Os desfechos de interesse do estudo foram aqueles relativos às infecções persistentes e lesões histológicas cervicais, por estarem diretamente relacionadas ao câncer do colo de útero. Lesões genitais externas não foram o foco principal desta revisão, porém, para o desfecho "infecção persistente ou doença” não foi possível realizar a análise dos dados em separado, já que a condição "doença" incluía tanto lesões externas quanto do colo do útero. Foram utilizados os seguintes desfechos: (i) infecção persistente; (ii) infecção persistente ou doença; (iii) neoplasia intraepitelial cervical de baixo grau/grau 2 (NIC 2); (iv) neoplasia intraepitelial cervical de alto grau/grau 3 (NIC 3); e (v) adenocarcinoma in situ.

Os desfechos relativos a "infecções persistentes” levaram em consideração dois períodos de duração - seis e 12 meses - e envolviam a detecção dos tipos de HPV relevantes para as vacinas em tela em duas avaliações consecutivas, sem negativação das amostras entre o intervalo de, no mínimo, cinco e 10 meses, respectivamente. Já o desfecho "infecção persistente ou doença" foi caracterizado por infecção pelo HPV mantida por seis meses ou mais, ou pela presença de doença cervical histológica ou genital externa. Os desfechos relativos à "neoplasia intracervical" (NIC 2 e NIC 3), bem como o de "adenocarcinoma in situ", tomaram por base o diagnóstico histológico destas condições.

Para a extração de dados, foi desenvolvido um formulário padrão com campos referentes às características de identificação dos estudos, características clínicas dos participantes, desfechos observados, medidas de eficácia e critérios da avaliação de qualidade.

Os dados foram armazenados por dupla digitação em um banco de dados do aplicativo EpiData (EpiData Assoc., Odense, Dinamarca).

\section{Análise estatística dos dados}

O tamanho do efeito da intervenção foi estimado usando-se a medida sumária do risco relativo (RR) e o respectivo intervalo de $95 \%$ de confiança (IC95\%). Um RR menor que um sugeria proteção vacinal contra o desfecho avaliado, com a eficácia sendo estimada como [1-RR] e expressa 
como porcentagem. Quando foi possível combinar resultados segundo diferentes desfechos clínicos, foram calculadas as medidas sumárias. Nessa análise, foi usado um modelo de efeitos aleatórios.

Foram realizados dois tipos de análise: por protocolo (de acordo com a intervenção realmente adotada durante o estudo) e, quando havia dados, também por intenção de tratar (como as pacientes foram designadas ao início do ensaio, tenham recebido a intervenção ou não).

Utilizou-se gráficos do tipo Forest na apresentação dos resultados da metanálise e comparação dos estudos.

A heterogeneidade foi medida pelo teste quiquadrado $\left(\chi^{2}\right)$ com significância assumida para $\mathrm{p}<0,1$. Outro método para avaliar a heterogeneidade entre os estudos foi pela inconsistência (I2), que descreve o porcentual de variação entre os estudos atribuída à heterogeneidade além da chance. Os valores da $I^{2}$ variam de $0-100 \%$. Assumiu-se que um valor de $0 \%$ era indicativo de não heterogeneidade e valores acima de $50 \%$ de heterogeneidade substancial.

Para as análises foi empregado o METAN, módulo específico para metanálises do Stata (Stata Corp., College Station, Estados Unidos).

\section{Resultados}

\section{Seleção dos estudos}

Foram identificados 378 estudos nas bases pesquisadas e dois por busca por referência cruzada. Após a eliminação de 53 duplicatas, os títulos restantes foram examinados, sendo excluídos 268 devido a: editoriais e cartas (1); estudos em animais (2); não atendimento dos critérios de inclusão, como: estudos não caracterizados como de eficácia; avaliação de vacinas não comercialmente disponíveis; vacinas monovalentes; amostra formada apenas por homens (265). Dos 59 trabalhos avaliados completamente, seis ensaios clínicos controlados randomizados foram incluídos na revisão sistemática 17,18,19,20,21,22. A Figura 1 sumariza os resultados das etapas de seleção e os motivos de exclusão.

\section{Características dos estudos e participantes}

Um total de 41.750 mulheres participou dos estudos selecionados, com a população por ensaio variando entre 552 e 18.644 mulheres, com faixa etária de 15 a 45 anos. Todos os trabalhos foram multinacionais, envolvendo múltiplos centros de 3 a 16 países das Américas do Norte e Latina, Europa e Ásia. Dois trabalhos incluíram pacientes brasileiras, correspondendo a $34 \%$ dos sujeitos em um 22 e $45 \%$ no outro ${ }^{18}$. Aproximadamente $90 \%$ das participantes dos ensaios apresentavam citologia normal no momento do recrutamento. Mulheres gestantes não fizeram parte de nenhuma população de estudo e cinco trabalhos excluíam, adicionalmente, participantes com história de exame citológico prévio com anormalidades. Exceto pelo estudo de Muñoz et al. 19, que não impunha restrições ao número de parceiros sexuais ao longo da vida, todos os demais incluíam participantes com menos de sete parceiros. As participantes foram acompanhadas nos ensaios com testagem semestral para DNA-HPV e com avaliação da presença de anormalidades citológicas a cada 6 a 12 meses. Em cinco dos seis estudos, o tempo médio do seguimento foi de três anos ou mais, e um destes alcançou cinco anos de tempo médio de acompanhamento. Foram incluídos quatro trabalhos sobre a vacina quadrivalente 17,19,21,22 e dois sobre a bivalente 18,20. Somente o estudo de Paavonen et al. 20 utilizou a vacina contra a hepatite A como comparador, o restante usou placebo. O esquema vacinal incluiu três doses, aplicadas com 0, 1 ou 2, e 6 meses. Todos os estudos incluídos tiveram como financiador uma das duas empresas farmacêuticas produtoras das vacinas comercialmente disponíveis (Tabela 1 ).

\section{Avaliação da qualidade metodológica} dos estudos

Apenas oito critérios do checklist CONSORT 16 não foram plenamente atendidos. Clareza quanto a objetivos específicos e hipóteses e descrição do fluxograma de pacientes estavam ausentes em dois trabalhos. Os procedimentos relativos à randomização e mascaramento foram adequados em todos os estudos; em apenas um 22 não estava descrito se a sequência de alocação foi mantida em sigilo até a intervenção. A descrição do cálculo do tamanho da amostra 19, relato da data de recrutamento e das datas do período de seguimento estiveram ausentes em um trabalho 22 . Em apenas um estudo 18 não havia relato das características clínicas dos grupos pesquisados, nem dos efeitos adversos apresentados pelos indivíduos 22 .

\section{Eficácia da vacina para o HPV segundo tipo de desfecho}

\section{- Eficácia na prevenção de infecção persistente}

Dois estudos 18,20 com vacina bivalente apresentaram resultados para estes desfechos - infecção 


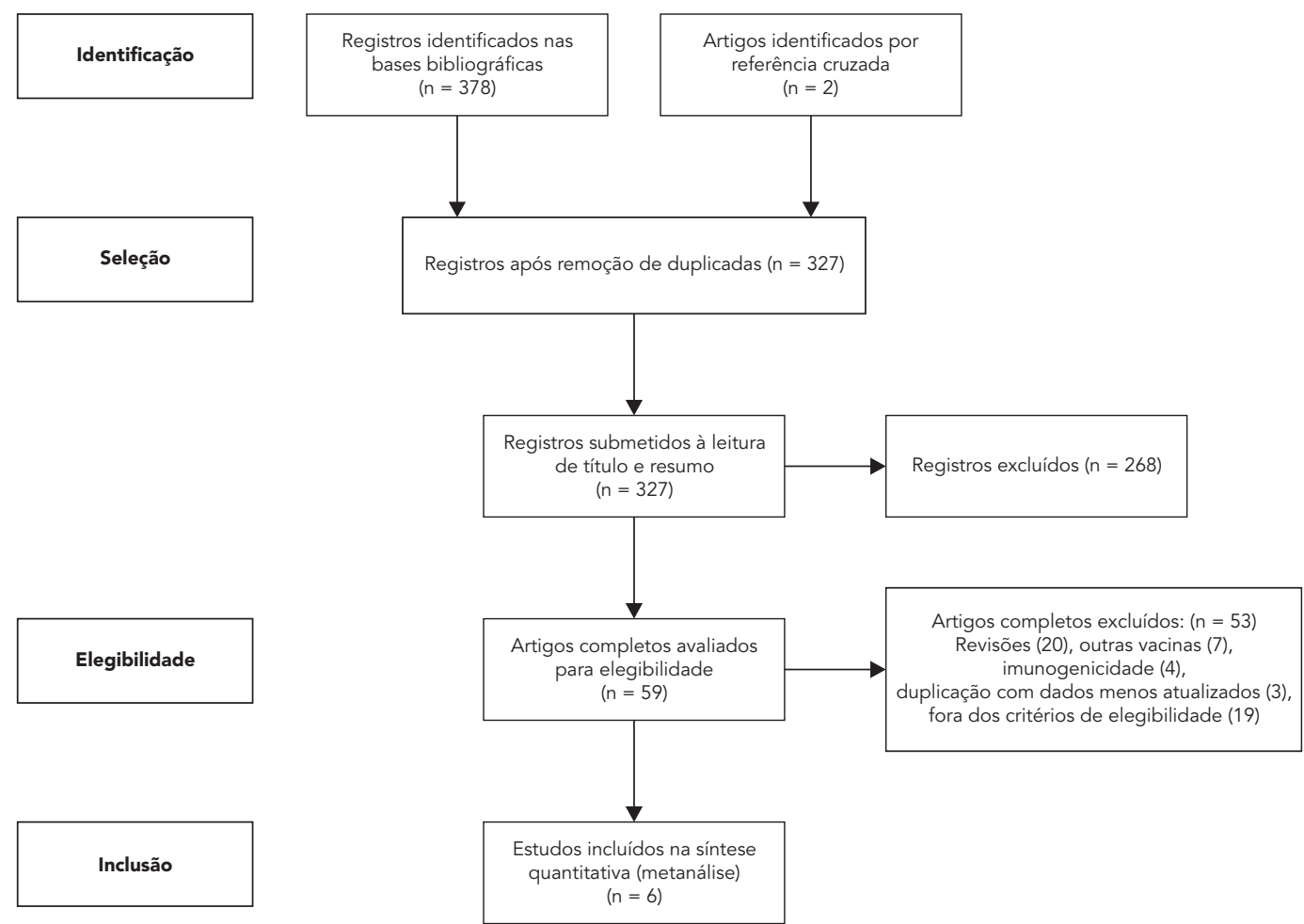

por seis e 12 meses - com as metanálises incluindo 15.098 mulheres, na faixa etária de 15 a 25 anos, ambas referindo-se apenas à análise por protocolo.

Para o desfecho relativo à persistência por seis meses, os resultados mostraram benefício estatisticamente significante com a vacinação (eficácia de 94\%, IC95\%: 91-96), sem heterogeneidade estatística entre os estudos $\left(\chi^{2}=0,2\right.$; $\mathrm{p}=0,658)$. No caso da persistência por $12 \mathrm{me}$ ses, a vacina forneceu proteção estatisticamente significante contra o HPV com eficácia de 91\% (IC95\%: 86-94), também sem heterogeneidade $\left(\chi^{2}=0,2 ; p=0,681\right)$.

\section{- Eficácia na prevenção de infecção persistente ou doença}

Dois trabalhos 19,22, ambos sobre vacina quadrivalente, apresentaram resultados para este desfecho, com tempo de seguimento de 26 a 60 meses (Figura 2).

Na metanálise, foram incluídas 4.371 mulheres de 16 a 45 anos. Os resultados de eficácia na avaliação segundo protocolo demonstraram diferença significante a favor do grupo de vacinados, correspondendo a uma eficácia de 93\%. Não houve heterogeneidade entre os estudos, com $\chi^{2}=0,9(p=0,353)$.

$\mathrm{Na}$ análise por intenção de tratar, ocorreram 112 eventos entre os 2.152 vacinados e 213 entre os 2.146 controles. Houve uma redução de 48\% (IC95\%: 35-58) na ocorrência de eventos no grupo que recebeu a vacina. Entretanto, houve evidência de heterogeneidade entre os estudos, $\operatorname{com} \chi^{2}=22,0(p=0,000)$.

\section{- Eficácia na prevenção de neoplasia intraepitelial cervical grau 2 (NIC 2)}

Três estudos, um com vacina bivalente 20 e dois com a quadrivalente 17,21 , apresentaram resultados para este desfecho, incluindo 29.720 mulheres, na faixa etária de 15 a 26 anos, com tempo de seguimento mais longo alcançando 36 meses (Figura 3).

A eficácia segundo análise por protocolo foi de 97\% (IC95\%: 90-99), sem heterogeneidade 
Características gerais dos estudos.

\begin{tabular}{|c|c|c|c|c|c|c|c|c|}
\hline Referência (ano) & $\begin{array}{l}\text { Duração } \\
\text { média de } \\
\text { seguimen- } \\
\text { to (meses) }\end{array}$ & $\begin{array}{c}\text { Grupo de } \\
\text { vacinação (n) }\end{array}$ & $\begin{array}{c}\text { Grupo de } \\
\text { comparação (n) }\end{array}$ & $\begin{array}{l}\text { Tipo de } \\
\text { vacina }\end{array}$ & $\begin{array}{l}\text { Idade em anos } \\
\text { (amplitude) }\end{array}$ & $\begin{array}{l}\text { Média de } \\
\text { idade no } \\
\text { grupo de } \\
\text { vacinação }\end{array}$ & $\begin{array}{c}\text { Média de } \\
\text { idade no grupo } \\
\text { de comparação }\end{array}$ & Desfechos avaliados \\
\hline $\begin{array}{l}\text { Harper et al. } 18 \\
(2006)\end{array}$ & 48 & 560 & 553 & Bivalente & $15-25$ & 20,4 & 20,5 & $\begin{array}{l}\text { Infecção incidental associada } \\
\text { ao HPV; Infecção persistente } \\
\text { por } 6 \text { meses; Infecção } \\
\text { persistente por } 12 \text { meses; } \\
\text { ASCUS; LBG (LSIL); NIC } 1 \\
\text { ou mais avançada; NIC } 2 \text { ou } \\
\text { mais avançada }\end{array}$ \\
\hline $\begin{array}{l}\text { Villa et al. } 22 \\
\text { (2006) }\end{array}$ & 60 & 277 & 275 & $\begin{array}{l}\text { Quadriva } \\
\text { lente }\end{array}$ & $16-23$ & 20,2 & 20,0 & $\begin{array}{l}\text { Infecção/doença associada } \\
\text { ao HPV; Doença associada } \\
\text { ao HPV; Infecção persistente } \\
\text { > } 12 \text { meses; NIC 1-3; } \\
\text { Condiloma }\end{array}$ \\
\hline $\begin{array}{l}\text { The FUTURE II } \\
\text { Study Group } 21 \\
\text { (2007) }\end{array}$ & 36 & 6.087 & 6.080 & $\begin{array}{l}\text { Quadriva } \\
\text { lente }\end{array}$ & $15-26$ & 20,0 & 19,9 & $\begin{array}{c}\text { NIC 2; NIC 3; } \\
\text { Adenocarcinoma in situ }\end{array}$ \\
\hline $\begin{array}{l}\text { Garland et al. } 17 \\
\text { (2007) }\end{array}$ & 36 & 2.723 & 2.732 & $\begin{array}{l}\text { Quadriva } \\
\text { lente }\end{array}$ & $16-24$ & 20,2 & 20,3 & $\begin{array}{c}\text { NIC 1; NIC 2; NIC 3; } \\
\text { Adenocarcinoma in situ }\end{array}$ \\
\hline $\begin{array}{l}\text { Muñoz et al. } 19 \\
\text { (2009) }\end{array}$ & 26 & 1.911 & 1.908 & $\begin{array}{l}\text { Quadriva } \\
\text { lente }\end{array}$ & $24-45$ & 34,3 & 34,3 & $\begin{array}{l}\text { Infecção ou doença associada } \\
\text { ao HPV; Doença associada ao } \\
\text { HPV; Infecção persistente > } \\
6 \text { meses }\end{array}$ \\
\hline $\begin{array}{l}\text { Paavonen et al. } 20 \\
\text { (2009) }\end{array}$ & 35 & 9.319 & 9.325 & Bivalente & $15-25$ & - & s & $\begin{array}{c}\text { Infecção associada por } 6 \\
\text { meses; Infecção associada } \\
\text { por } 12 \text { meses; NIC } 2 \text { ou mais } \\
\text { avançada; NIC } 3 \text { ou mais } \\
\text { avançada }\end{array}$ \\
\hline
\end{tabular}

ASCUS: atipias de significado indeterminado em células escamosas; HPV: papilomavirus humano; LBG: lesão intraepitelial de baixo grau;

NIC1: lesão intraepitelial de grau 1; NIC 2: lesão intraepitelial de grau 2; NIC 3: lesão intraepitelial de grau 3.

entre os resultados, $\chi^{2}=0,4(p=0,813)$. Entretanto, na análise por intenção de tratar, a eficácia estimada foi bem mais baixa (63\%, IC95\%: 52 $71)$, com presença de heterogeneidade $\left(\chi^{2}=22,7\right.$; $\mathrm{p}=0,000)$.

Quando considerados apenas os estudos da vacina quadrivalente, a análise por protocolo indicou eficácia vacinal de 98\% (IC95\%: 86-100), sem heterogeneidade. Já na análise por intenção de tratar, a eficácia foi de apenas 48\% (IC95\%: 31-60), com presença de heterogeneidade $\left(\chi^{2}=3,17 ; p=0,075\right)$.

\section{- Eficácia na prevenção da neoplasia intraepitelial cervical grau 3 (NIC 3)}

Três trabalhos ( $\mathrm{n}=29.720,15$ a 26 anos), um com vacina bivalente 20 e dois com a quadrivalen- te 17,21 , apresentaram resultados para este desfecho.

Os resultados (Figura 4) apontam para uma eficácia vacinal na análise por protocolo de $96 \%$ (IC95\%: 89-99), com homogeneidade entre os resultados dos estudos $\left(\chi^{2}=0,08 ; p=0,960\right)$. A eficácia na análise por intenção de tratar foi inferior, $42 \%$ (IC95\%: 26-55) e foi detectada heterogeneidade $\left(\chi^{2}=10,26 ; p=0,006\right)$.

Metanálise somente com os dois estudos da vacina quadrivalente indicou $97 \%$ de eficácia na análise por protocolo, com diferença significante a favor do grupo de vacinados (IC95\%: 84-99). Nesse caso, o teste $\left(\chi^{2}=0,01\right)$ rejeitou heterogeneidade, com valor de p igual a 0,922. Na análise por intenção de tratar, a eficácia foi de $35 \%$ (IC95\%: 6-50), com presença de heterogeneidade $\left(\chi^{2}=3,16 ; p=0,075\right)$. 
Figura 2

Eficácia da vacina quadrivalente na prevenção da ocorrência de infecção persistente ou doença.

2a) Análise por protocolo

\begin{tabular}{|c|c|c|c|}
\hline Estudo & & RR (IC95\%) & Peso (\%) \\
\hline Villa et al. ${ }^{22}$ & $\longleftrightarrow$ : & $0,04(0,01-0,18)$ & 52,92 \\
\hline Muñoz et al. ${ }^{19}$ & $!$ & $0,10(0,03-0,27)$ & 47,08 \\
\hline Medida combinada $\left(l^{2}=0,0 \% ; p=0,353\right)$ & $>$ & $0,07(0,03-0,16)$ & 100,00 \\
\hline
\end{tabular}

2b) Análise por intenção de tratar

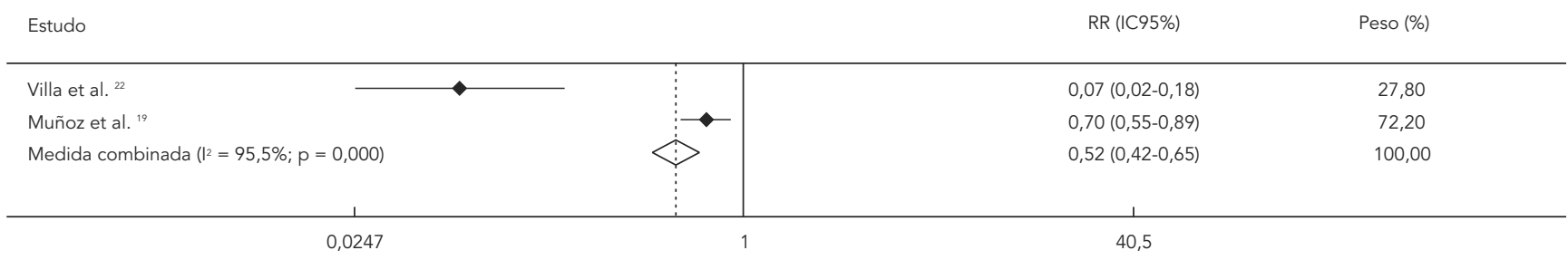

IC95\%: intervalo de 95\% de confiança; RR: risco relativo.

Figura 3

Eficácia das vacinas quadrivalente e bivalente na prevenção da ocorrência de NIC 2.

3a) Análise por protocolo

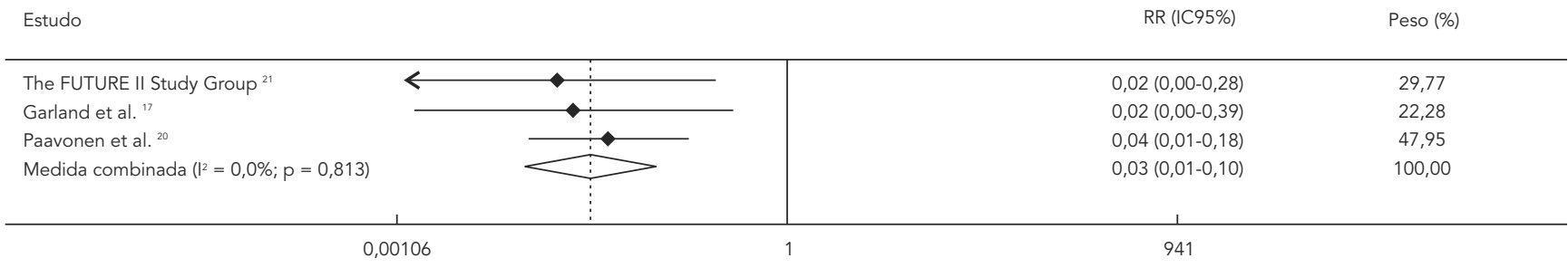

3b) Análise por intenção de tratar

Estudo

RR (IC95\%) Peso (\%)

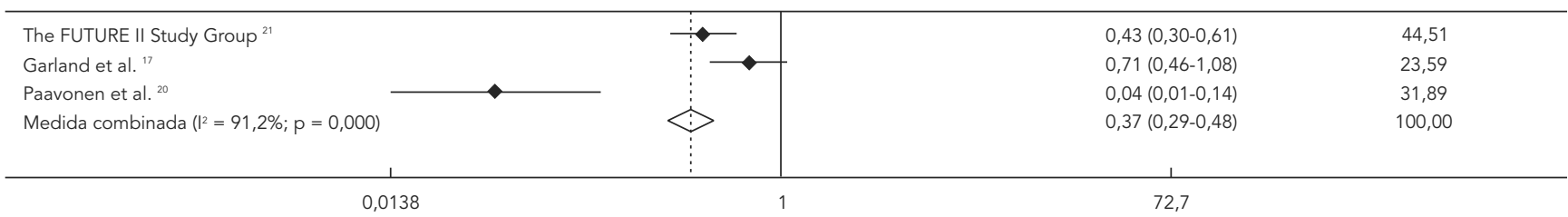

IC95\%: intervalo de 95\% de confiança; NIC2: lesão intraepitelial de grau 2; RR: risco relativo. 


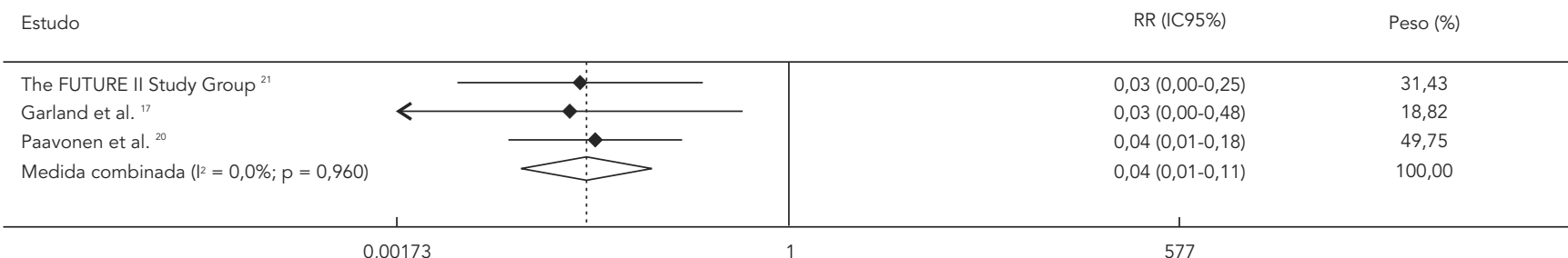

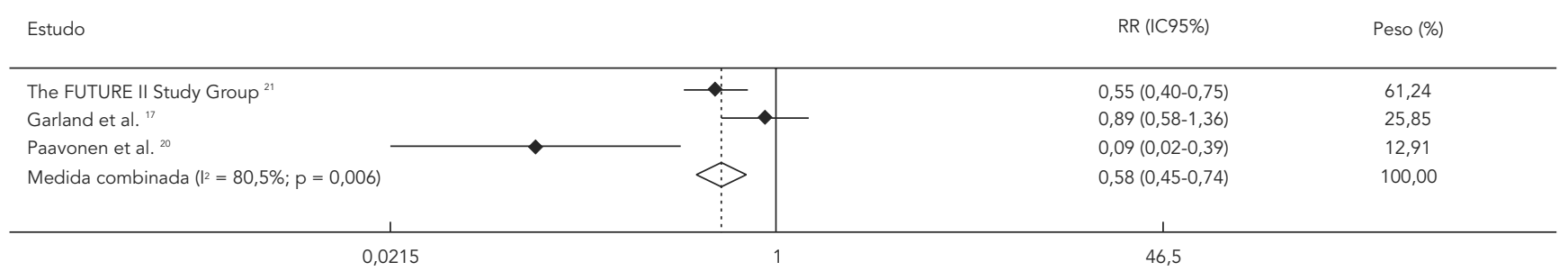

IC95\%: intervalo de 95\% de confiança; NIC3: lesão intraepitelial de grau 3; RR: risco relativo.

\section{- Eficácia na prevenção do adenocarcinoma in situ}

Dois estudos com vacina quadrivalente 17,21 avaliaram a ocorrência de adenocarcinoma, com o maior tempo de seguimento sendo inferior a 36 meses. Na metanálise, foram incluídas 17.622 mulheres com idades entre 15 e 26 anos.

A avaliação por protocolo não permitiu concluir sobre a eficácia da vacina para HPV: o risco relativo combinado foi de 0,13 (IC95\%: 0,02-1,00), mas o intervalo de confiança da medida sumária incluiu o valor nulo. Na análise por intenção de tratar, o RR combinado foi de 0,46 (IC95\%: 0,181,21), também sem significância estatística.

\section{Discussão}

Esta revisão permitiu examinar as evidências estratificadas por tipo de desfecho, apresentando a eficácia vacinal separadamente para persistência da infecção pelo HPV, lesões intraepiteliais de graus 2 e 3 , e adenocarcinoma. No caso do desfecho lesão intraepitelial de grau 2, a eficá- cia foi próxima a 98\%, tanto para as vacinas examinadas conjuntamente quanto para a vacina quadrivalente tomada de forma isolada. Para as lesões de grau 3, a eficácia também foi alta. Assim, a revisão indicou que as vacinas comercialmente disponíveis contra a infecção pelo HPV são eficazes em mulheres que não tenham infecção prévia pelos subtipos virais constituintes dos imunobiológicos estudados, considerando esses desfechos intermediários. Contudo, no caso do adenocarcinoma in situ, embora as estimativas pontuais da eficácia vacinal sinalizassem tendência de proteção, os resultados não foram estatisticamente significantes, seja na avaliação por protocolo, seja por intenção de tratar.

Um aspecto importante de ser destacado refere-se às eficácias vacinais bem menores nas análises por intenção de tratar, relativos a todos os desfechos analisados. A análise por intenção de tratar parte do pressuposto que os desvios do protocolo inicial são irrelevantes frente à garantia de comparabilidade dos grupos obtida pela randomização. Outrossim, essa abordagem de análise reflete uma avaliação do benefício frente a perdas ou mudanças ocasionais na orientação 
de tratamento previsto no protocolo inicial, como por exemplo, a incompletude do esquema vacinal proposto, passível de acontecer em uma intervenção que se apoia em doses repetidas em uma população que usualmente é menos acessível a um cuidado regular. Como a análise por intenção de tratar garante a randomização, o achado pode indicar que as estimativas de eficácia para a abordagem por protocolo poderiam estar enviesadas. De forma semelhante a este trabalho, revisões sistemáticas realizadas por Rambout et al. 14 e por Lu et al. 23 também apresentaram resultados por intenção de tratar com eficácia menor que nas análises por protocolo.

A eficácia na prevenção do câncer do colo de útero, enquanto desfecho finalístico observado apenas no longo prazo, persiste como uma questão a ser respondida. Considerando a história natural da doença, o efeito da imunização na incidência da neoplasia somente poderá ser sentido após um longo período de latência, que se estima superior a pelo menos 10-20 anos após a introdução de um programa de vacinação 24 . Os estudos clínicos controlados disponíveis têm tempo de seguimento curto, que não ultrapassa 60 meses, e apenas indicam eficácia relativa a resultados considerados intermediários na história natural da doença, como a redução da infecção persistente ou de lesões precursoras do câncer cérvico-uterino. Assim, embora os achados possam indicar diminuição na propensão ao desenvolvimento do desfecho definitivo (no caso, o câncer do colo de útero), sinalizando para resultados promissores, deve-se levar em consideração que parte dessas lesões regride, em alguns casos mesmo sem tratamento. Östor 25 , em uma revisão de trabalhos que avaliaram a história natural das lesões intraepiteliais cervicais, publicados entre 1950 e 1990, concluiu que a probabilidade de um epitélio atípico evoluir para neoplasia invasora é diretamente proporcional à gravidade da atipia; todavia, esta progressão não é irreversível, ocorrendo com frequência regressão das lesões para estágios anteriores. Os resultados desse autor apontam que, no caso das lesões intraepiteliais de grau $2,42 \%$ regridem, $40 \%$ persistem e a progressão para invasão ocorre apenas em 5\%. Já nas lesões intraepiteliais de grau 3, embora a progressão para invasão seja superior a $12 \%$, as lesões regridem espontaneamente em aproximadamente um terço dos casos. Outros trabalhos sinalizam no mesmo sentido, com a probabilidade de regressão das lesões de grau 3 menor que as de segundo e uma maior propensão de evolução da doença para câncer do colo de útero 26,27.

Podem existir variações locais da prevalência específica dos subtipos virais do HPV nos diver- sos países e até continentes 28,29, o que justificaria possíveis diferenças na efetividade das vacinas. Ainda que os subtipos virais 16 e 18 sejam os mais frequentes, inclusive na América Latina e Caribe 30, os subtipos 31, 33, 35, 45, 52 e 58 são responsáveis por 20 a $30 \%$ dos casos de câncer do colo de útero em todo o mundo. Nesse sentido, as conclusões acerca da eficácia vacinal de qualquer revisão sistemática, inclusive esta, apenas deverão ser generalizadas se considerada a prevalência específica dos subtipos virais.

Sabe-se que o risco de infecção pelo HPV aumenta com a idade 31,32 , e a vacinação, de caráter preventivo, é preconizada de ser realizada em faixas etárias precoces, no caso adolescentes e pré-adolescentes, de modo a imunizá-las antes da vida sexual ativa. Uma suposição que se pretendia examinar, quando da realização desta metanálise, era que a eficácia das vacinas vem sendo testada em mulheres com faixa etária diferente daquela indicada como o principal grupo alvo, qual seja, meninas entre 9 e 15 anos antes da sexarca. Todos os estudos identificados corroboram nesse sentido, pois as populações estudadas eram de mulheres com idade mínima de 15 anos, indo até os 45, isto é, fora da população alvo. Não foi possível, contudo, estratificar os resultados por idades dentro dessa faixa mais ampla, dado que apenas um estudo (Munoz 19) trazia resultados em subgrupos de idade.

Trabalhos com populações mais jovens estão focados, geralmente, em avaliar a presença de imunogenicidade 33,34. Eles têm por pressuposto que a presença e persistência de anticorpos neutralizantes nas superfícies mucosas possam ser um fator determinante de proteção, ainda que seja desconhecida a velocidade de declínio destes anticorpos e a necessidade de doses de reforço subsequente, com seus impactos nos custos da estratégia de prevenção primária. Desse modo, não é possível afirmar que os resultados de eficácia verificados nesta revisão possam ser seguramente generalizados para a população alvo. A lacuna verificada assinala a necessidade de mais estudos, com foco na avaliação dos grupos etários mais jovens, uma vez que as formulações vacinais já foram aprovadas e estão sendo usadas de forma sistemática em alguns países.

A eficácia das vacinas para o HPV foi objeto de outras revisões sistemáticas e metanálises 12,13,14,23,35,36,37, as quais também indicam um efeito protetor da imunização profilática em mulheres sem exposição prévia aos subtipos virais. Os desfechos utilizados nesses estudos de síntese variaram significativamente, dificultando a comparação de seus achados com os nossos resultados. 
Cabe ainda serem assinaladas algumas outras diferenças em relação a esses trabalhos. Nossa revisão estendeu o período de busca bibliográfica até novembro de 2009, o que ultrapassa inclusive o período da revisão de Lu et al. ${ }^{23}$, que encerrou em julho de 2009. Embora a nossa busca compreenda um período anterior àquele do estudo de síntese publicado por Rey-Ares et al. ${ }^{37}$, que foi até julho de 2011, o número de estudos incluídos por esses autores foi menor (apenas quatro trabalhos) do que o nosso. Na revisão de Rey-Ares não foram incluídos os estudos de Villa et al. 22, de 2006, e de Garland et al. 17, de 2007. O estudo Future II 21, também do mesmo ano, foi substituído por Munoz et al. 38, de 2010, que não estava disponível quando da busca realizada para o nosso trabalho. Em contrapartida, nós incluímos um outro estudo de Munoz et al. 19: uma avaliação multicêntrica randomizada da vacina quadrivalente, envolvendo 3.817 mulheres de 24 a 45 anos, sem história prévia de úlceras genitais ou de doença cervical e soronegativas para os subtipos vacinais no recrutamento. Esse trabalho é o único que traz dados estratificados por faixa etária. No caso da faixa de 24 a 34 anos, a eficácia da vacina foi de 92\% (IC95\%: 67-99), enquanto para as mulheres de 35 a 45 anos foi de 89\% (IC95\%: 52-99).

É importante ter em mente que as vacinas para HPV são ainda bastante recentes e que o número de trabalhos e o tempo de seguimento dos estudos primários são ainda pequenos, sendo identificadas várias publicações relativas às mesmas populações de estudo, mas trazendo diferentes tempos de seguimento, o que reforça a importância de monitoramento de novos trabalhos com tempos maiores de acompanhamento.

Exceto pelo nosso trabalho e o de Rey-Ares et al. 37 , todos os demais incluíam a vacina monovalente. Esse imunobiológico não está comercialmente disponível para uso, e os estudos, geralmente de fase I ou II, foram usualmente realizados para avaliação de imunogenicidade. A diferença entre esta metanálise e a de Lu et al. 23, que incluiu sete estudos, repousa exatamente na inclusão de um ensaio com vacina monovalente não comercializada, o trabalho de Koutsky et al. 39 . Tratou-se, no nosso caso, de uma opção metodológica assumida pelos autores, entendendo que o escopo da revisão deveria privilegiar a aplicabilidade de seus resultados em termos de uso populacional.

Os resultados promissores apontados não eliminam a necessidade da continuidade da realização de rastreamento regular pelo teste de Papanicolaou, que tem mostrado custo-efetivo em nosso país 40 desde que mantenha elevada cobertura populacional e adequada indicação e realização do exame, com encaminhamento ao tratamento correto e oportuno, nos casos necessários. Cerca de 30\% dos tipos virais oncogênicos não são cobertos pelas vacinas atualmente disponíveis; portanto, mesmo vacinadas, as mulheres podem ser infectadas por subtipos virais não constituintes dos imunobiológicos. A vacina, ademais, tem ação preventiva; imuniza os indivíduos contra a infecção por HPV, mas não erradica a infecção já existente. Por conseguinte, mulheres já infectadas necessitam ser rastreadas e tratadas para impedir que suas lesões progridam, o que por si só já inviabiliza pensar que a vacinação tornará os presentes programas de rastreamento desnecessários.

O pequeno número de estudos incluídos é uma limitação nas evidências apontadas por esta revisão. Entretanto, as outras revisões sistemáticas anteriormente referidas esbarram na mesma escassez, que reflete a ainda pequena produção científica na área. À proporção que novos estudos sejam publicados, estes poderão ser agregados e a metanálise poderá ser atualizada.

Outra limitação foi a impossibilidade da avaliação do viés de publicação, devido ao número muito baixo de trabalhos incluídos por desfecho. Entretanto, a existência de outras revisões sistemáticas com semelhante número de artigos incluídos reforça a baixa plausibilidade de viés desse tipo e indica o atual estágio desse programa de investigação.

A ocorrência de viés de idioma é pouco provável, já que não foram identificados artigos potencialmente relevantes não publicados em inglês, espanhol ou português, não havendo exclusão de qualquer publicação por este motivo.

Esta revisão não pretendeu avaliar a eficácia vacinal contra úlceras anogenitais e outras lesões vaginais ou vulvares associadas ao HPV. Essas diversas lesões podem ser prevenidas com a vacina quadrivalente, que apresenta adicionalmente os subtipos virais 6 e 11, com relatos na literatura de eficácia vacinal atingindo até $90 \%$ 8,17.

Por fim, um último aspecto a ser levado em conta na avaliação da eficácia é a possibilidade de conflitos de interesse, já que todos os estudos originais foram apoiados pelas grandes empresas fabricantes das vacinas. Sabe-se dos interesses financeiros envolvidos no desenvolvimento e comercialização de novas tecnologias em saúde e do poder que as indústrias produtoras têm de influenciar na pesquisa médica e prática clínica. Angell 41, desde 2007, vem sinalizando que os resultados das pesquisas financiadas pela indústria favorecem os seus produtos, quando comparados a trabalhos com outras fontes de financiamento. Uma revisão realizada recentemente apoia a existência dessa influência, que inclui a 
produção e sínteses das evidências, estudos de custo-efetividade, guias de práticas e, também, as decisões de profissionais e consumidores 42 .

Em conclusão, a vacinação profilática parece prevenir a infecção pelo HPV em mulheres de 15 a 45 anos sem infecção prévia pelos subtipos virais por pelo menos cinco anos. Entretanto, a ausência de períodos de seguimento mais longos torna impossível definir a eficácia de longo prazo dessa estratégia de prevenção primária, sendo ainda cedo para avaliar seu impacto na incidência e mortalidade pelo câncer do colo de útero.

\section{Resumen}

La infección persistente por VPH es una condición necesaria para la aparición de cáncer del cuello del útero. Con el fin de reducir la incidencia se han desarrollado vacunas profilácticas contra el VPH, existiendo dos formulaciones disponibles comercialmente: bivalentes (tipos 16, 18) y tetravalente $(6,11,16$ y 18). Se realizó un meta-análisis de la eficacia de estas vacunas en las mujeres, centrándose en la evaluación estratificada por los resultados clínicos. Se identificaron ensayos controlados aleatorios (ECA) publicados entre 2000 y 2009 en las basis MEDLINE, LILACS y Cochrane Library, y evaluados por dos revisores independientes. Seis ECA fueron incluidos. La vacuna reduce el riesgo de las lesiones precursoras del cáncer, con una eficacia de 97\% (IC95\%: 90-99) para CIN 2 y 96\% (IC95\%: 89-99) para CIN 3, en el análisis por protocolo. La eficiencia en el análisis por intención de tratar fue menor: 63\% (IC95\%: 52-71) y 42\% (IC95\%: 26-55), respectivamente. Para evaluar su efectividad en la incidencia y mortalidad del cáncer cervical, se necesitan estudios con un seguimiento más prolongado.

Vacunas contra Papillomavirus; Neoplasias del Cuello Uterino; Eficacia; Evaluación de Tecnologías de Salud

\section{Colaboradores}

S. C. F. Araujo, R. Caetano e J. U. Braga participaram da concepção, planejamento, análise, interpretação dos dados, elaboração do rascunho, revisão crítica do conteúdo, e aprovação da versão final do trabalho. F. V. Costa e Silva participou da coleta e análise dos dados e aprovação da versão final do trabalho.

\section{Agradecimentos}

Este trabalho fez parte de uma dissertação de mestrado profissional na área de Gestão de Tecnologias em Saúde, realizado com o financiamento do Departamento de Ciência e Tecnologia do Ministério da Saúde.

\section{Conflito de interesses}

Não declarado. 


\section{Referências}

1. Castellsagué X, De SanJose T, Aguado KS, Louie L, Bruni J, Muñoz M. HPV and cervical cancer in the 2007 report. Vaccine 2007; 25 Suppl 3:C1-230.

2. Ferlay J, Shin HR, Bray F, Forman D, Mathers C, Parkin DM. Estimates of worldwide burden of cancer in 2008: GLOBOCAN 2008. Int J Cancer 2010; 127:2893-917.

3. Coordenação de Prevenção e Vigilância, Coordenação Geral de Ações Estratégicas, Instituto Nacional de Câncer José Alencar Gomes da Silva. Estimativa 2012: incidência de câncer no Brasil. Rio de Janeiro: Instituto Nacional de Câncer José Alencar Gomes da Silva; 2011.

4. Walboomers JM, Jacobs MV, Manos MM, Bosch FX, Kummer JA, Shah KV, et al. Human papillomavirus is a necessary cause of invasive cervical cancer worldwide. J Pathol 1999; 189:12-9.

5. Parkin DM, Bray F, Ferlay J, Pisani P. Global cancer statistics, 2002. CA Cancer J Clin 2005; 55:74-108.

6. Ho GY, Bierman R, Beardsley L, Chang CJ, Burk RD. Natural history of cervicovaginal papillomavirus infection in young women. N Engl J Med 1998; 338:423-8.

7. Garland SM. Can we really beat cervical cancer? Med J Aust 2003; 178:647-9.

8. FUTURE I/II Study Group; Dillner J, Kjaer SK, Wheeler CM, Sigurdsson K, Iversen OE, et al. Four year efficacy of prophylactic human papillomavirus quadrivalent vaccine against low grade cervical, vulvar, and vaginal intraepithelial neoplasia and anogenital warts: randomised controlled trial. BMJ 2010; 20:c3493.

9. Munoz N, Bosch FX, de Sanjose S, Herrero R, Castellsague X, Shah KV, et al. Epidemiologic classification of human papillomavirus types associated with cervical cancer. N Engl J Med 2003; 348: 518-27.

10. Sawaya GF, Smith-McCune K. HPV vaccination: more answers, more questions. N Engl J Med 2007; 356:1991-3.

11. Munoz N, Franco EL, Herrero R, Andrus JK, de Quadros C, Goldie SJ, et al. Recommendations for cervical cancer prevention in Latin America and the Caribbean. Vaccine 2008; 26 Suppl 11:L96-107.

12. La Torre G, de Waure C, Chiaradia G, Mannocci A, Ricciardi W. HPV vaccine efficacy in preventing persistent cervical HPV infection: a systematic review and meta-analysis. Vaccine 2007; 25:8352-8.

13. Medeiros LR, Rosa DD, da Rosa MI, Bozzetti MC, Zanini RR. Efficacy of human papillomavirus vaccines: a systematic quantitative review. Int J Gynecol Cancer 2009; 19:1166-76.

14. Rambout L, Hopkins L, Hutton B, Fergusson D. Prophylactic vaccination against human papillomavirus infection and disease in women: a systematic review of randomized controlled trials. CMAJ 2007; 177:469-79.

15. Haynes RB, Wilczynski N, McKibbon KA, Walker CJ, Sinclair JC. Developing optimal search strategies for detecting clinically sound studies in MEDLINE. J Am Med Inform Assoc 1994; 1:447-58.
16. Altman DG, Schulz KF, Moher D, Egger M, Davidoff F, Elbourne D, et al. The revised CONSORT statement for reporting randomized trials: explanation and elaboration. Ann Intern Med 2001; 134: 663-94.

17. Garland SM, Hernandez-Avila M, Wheeler CM, Perez G, Harper DM, Leodolter S, et al. Quadrivalent vaccine against human papillomavirus to prevent anogenital diseases. N Engl J Med 2007; 356:1928-43.

18. Harper DM, Franco EL, Wheeler CM, Moscicki AB, Romanowski B, Roteli-Martins CM, et al. Sustained efficacy up to 4.5 years of a bivalent L1 virus-like particle vaccine against human papillomavirus types 16 and 18: follow-up from a randomised control trial. Lancet 2006; 367:1247-55.

19. Munoz N, Manalastas Jr. R, Pitisuttithum P, Tresukosol D, Monsonego J, Ault K, et al. Safety, immunogenicity, and efficacy of quadrivalent human papillomavirus (types $6,11,16,18$ ) recombinant vaccine in women aged $24-45$ years: a randomised, double-blind trial. Lancet 2009; 373:1949-57.

20. Paavonen J, Naud P, Salmeron J, Wheeler CM, Chow SN, Apter D, et al. Efficacy of human papillomavirus (HPV)-16/18 AS04-adjuvanted vaccine against cervical infection and precancer caused by oncogenic HPV types (PATRICIA): final analysis of a double-blind, randomised study in young women. Lancet 2009; 374:301-14.

21. The FUTURE II Study Group. Quadrivalent vaccine against human papillomavirus to prevent high-grade cervical lesions. N Engl J Med 2007; 356:1915-27.

22. Villa LL, Costa RL, Petta CA, Andrade RP, Paavonen J, Iversen OE, et al. High sustained efficacy of a prophylactic quadrivalent human papillomavirus types 6/11/16/18 L1 virus-like particle vaccine through 5 years of follow-up. Br J Cancer 2006; 95:1459-66.

23. Lu B, Kumar A, Castellsague X, Giuliano AR. Efficacy and safety of prophylactic vaccines against cervical HPV infection and diseases among women: a systematic review \& meta-analysis. BMC Infect Dis 2011; 11:13.

24. Kulasingam SL, Benard S, Barnabas RV, Largeron N, Myers ER. Adding a quadrivalent human papillomavirus vaccine to the UK cervical cancer screening programme: a cost-effectiveness analysis. Cost Eff Resour Alloc 2008; 6:4.

25. Ostor AG. Natural history of cervical intraepithelial neoplasia: a critical review. Int J Gynecol Pathol 1993; 12:186-92.

26. ASCUS-LSIL Traige Study (ALTS) Group. Results of a randomized trial on the management of cytology interpretations of atypical squamous cells of undetermined significance. Am J Obstet Gynecol 2003; 188:1383-92.

27. Wright Jr. TC, Cox JT, Massad LS, Twiggs LB, Wilkinson EJ. 2001 consensus guidelines for the management of women with cervical cytological abnormalities. JAMA 2002; 287:2120-9. 
28. Bosch FX, Manos MM, Munoz N, Sherman M, Jansen AM, Peto J, et al. Prevalence of human papillomavirus in cervical cancer: a worldwide perspective. International Biological Study on Cervical Cancer (IBSCC) Study Group. J Natl Cancer Inst 1995; 87:796-802.

29. Clifford G, Franceschi S, Diaz M, Muñoz N, Villa LL. Chapter 3: HPV type-distribution in women with and without cervical neoplastic diseases. Vaccine 2006; 24 Suppl 3:S3/26-34.

30. Ciapponi A, Bardach A, Glujovsky D, Gibbons L, Picconi MA. Type-specific HPV prevalence in cervical cancer and high-grade lesions in Latin America and the Caribbean: systematic review and meta-analysis. PLoS One 2011; 6:e25493.

31. Castle PE, Schiffman M, Herrero R, Hildesheim A, Rodriguez AC, Bratti MC, et al. A prospective study of age trends in cervical human papillomavirus acquisition and persistence in Guanacaste, Costa Rica. J Infect Dis 2005; 191:1808-16.

32. Winer RL, Koutsky LA. Human papillomavirus through the ages. J Infect Dis 2005; 191:1787-9.

33. Giuliano AR, Lazcano-Ponce E, Villa L, Nolan T, Marchant C, Radley D, et al. Impact of baseline covariates on the immunogenicity of a quadrivalent (types 6, 11, 16, and 18) human papillomavirus virus-like-particle vaccine. J Infect Dis 2007; 196:1153-62.

34. Kang S, Kim KH, Kim YT, Kim YT, Kim JH, Song YS, et al. Safety and immunogenicity of a vaccine targeting human papillomavirus types $6,11,16$ and 18: a randomized, placebo-controlled trial in 176 Korean subjects. Int J Gynecol Cancer 2008; 18:1013-9.

35. Kjaer SK, Sigurdsson K, Iversen OE, HernandezAvila M, Wheeler CM, Perez G, et al. A pooled analysis of continued prophylactic efficacy of quadrivalent human papillomavirus (Types 6/11/16/18) vaccine against high-grade cervical and external genital lesions. Cancer Prev Res (Phila) 2009; 2: 868-78.
36. McCormack PL, Joura EA. Quadrivalent human papillomavirus (types $6,11,16,18$ ) recombinant vaccine (Gardasil $\AA$ ): a review of its use in the prevention of premalignant genital lesions, genital cancer and genital warts in women. Drugs 2010; 70:2449-74.

37. Rey-Ares L, Ciapponi A, Pichon-Riviere A. Efficacy and safety of human papilloma virus vaccine in cervical cancer prevention: systematic review and meta-analysis. Arch Argent Pediatr 2012; 110:483-9.

38. Munoz N, Kjaer SK, Sigurdsson K, Iversen OE, Hernandez-Avila M, Wheeler CM, et al. Impact of human papillomavirus (HPV)-6/11/16/18 vaccine on all HPV-associated genital diseases in young women. J Natl Cancer Inst 2010; 102:325-39.

39. Koutsky LA, Ault KA, Wheeler CM, Brown DR, Barr E, Alvarez FB, et al. A controlled trial of a human papillomavirus type 16 vaccine. N Engl J Med 2002; 347:1645-51.

40. Caetano R, Vianna CMM, Thuler LC, Girianelli V. Custo-efetividade no diagnóstico precoce do câncer de colo uterino no Brasil. Physis (Rio J.) 2006; 16:99-118.

41. Angell M. A verdade sobre os laboratórios farmacêuticos. São Paulo: Record; 2007.

42. Stamatakis E, Weiler R, Ioannidis JP. Undue industry influences that distort healthcare research, strategy, expenditure and practice: a review. Eur J Clin Invest 2013; 43:469-75.

Recebido em 13/Nov/2012

Versão final reapresentada em 05/Mai/2013

Aprovado em 04/Jul/2013 